\title{
Collaboration among Crowdsourcees: Towards a Design Theory for Collaboration Process Design
}

\author{
Navid Tavanapour \\ Information Systems, Universität Hamburg \\ tavanapour@,informatik.uni-hamburg.de
}

\author{
Eva A. C. Bittner \\ Information Systems, Universität Hamburg \\ bittner@informatik.uni-hamburg.de
}

\begin{abstract}
Crowdsourcing is used for collaborative problem solving in different domains. The key to optimal solutions is mostly found by collaboration among the crowdsourcees. The current state of research on this field addresses this topic mainly with an explorative focus on a specific domain, such as idea contests. We gather and analyze the contributions from the different domains on collaboration in crowdsourcing. We present a framework for a general collaboration process model for crowdsourcing. To derive this framework, we conducted a literature review and set up a database, which assigns the literature to the process steps that we identified from interaction patterns in the literature. The framework considers phases before and after the collaboration among crowdsourcees and includes relevant activities that can influence the collaboration process. This paper contributes to a deeper understanding of the interaction among crowdsourcees and provides crowdsourcers with grounding for the informed design of effective collaborative crowdsourcing processes.
\end{abstract}

\section{Introduction}

Crowdsourcing includes tasks and relates to the process of "outsourcing it to an undefined, generally large group of people in an open call" [13]. Howe (2008) describes it as a way for organizations to expand their human resources and the work force, which increasingly finds interest in corporate practice [19]. The core of outsourcing is to use the collective intelligence of the crowd for solving problems involved in tasks [14]. Some tasks can be broken down into subtasks and edited independently by individuals from the crowd. For some tasks the collaboration of several crowdsourcees is required. We use the term "crowdsourcee" for individuals of the crowd, who respond to an open call and the term "crowdsourcer" for proposers of an open call [7]. In particular for complex tasks, which exceed the capacity and skills of individuals, collaboration of heterogeneous actors has the potential to lead to better results, if orchestrated well $[4,5,18,26]$. Research in this field identified the collaboration among crowdsourcees to have qualitative impact on the outcome of the task accomplishment [15] and as a main part for value creation [1]. However, collaboration among crowdsourcees has been identified as a research gap in prior research, especially the understanding of the collaborative interaction between crowdsourcees [1]. Extant literature either presents a general view on crowdsourcing and refers to collaboration only shortly on a global level, e.g. the review of [23], or they use explorative approaches to analyze scenarios in specific domains. In this emergent stream of literature, our attempt is to understand the mechanisms, processes and challenges in crowdsourcee collaboration for effective collaborative crowdwork practices. Therefore, we consolidate the current research on this field and put together the relevant findings of prior studies in a framework. We introduce a generalized collaboration process for collaborative crowdsourcing. This serves to understand the collaboration among crowdsourcees and supports crowdsourcers to build structured and goal oriented effective collaborative processes into their crowdsourcing initiatives. To do so this paper represents a review of the nascent literature on collaboration in crowdsourcing to illuminate the current state of this so far under-explored topic.

The review is guided by the following research questions: How are the collaboration processes between crowdsourcees structured? What indicators can be identified to reconstruct the collaboration process among crowdsourcees? This paper builds on prior concepts (such as [23]) and deepens the focus to the collaboration process and relevant process indicators in the literature.

The structure of this paper is as follows: First, the methodology for the systematic integrative literature review conducted will be presented. Second, this review compares the considered literature according to their domain and the contribution they deliver 
considering the methodologies used and their type of contributions. Third, we use the literature review as basis to identify the status quo of research on collaboration between crowdsourcees. Forth, we give an overview on the different phases and collaborative activities that are revealed in the papers. Fifth, we structure our findings and introduce a framework for the collaboration process of the interaction among crowdsourcees.

Finally, we close this paper with a conclusion and outlook on future research.

\section{Methodology}

This paper presents the state of research on collaboration processes among crowdsourcees by first, conducting a systematic literature review. We follow the descriptions of [6] for the comprehensive search process of the literature, to ensure the completeness and the thoroughness of this review.

Initially, we considered the databases IEEE Xplore Digital Library, Ebscohost research database, ACM Digital Library, Emerald insight, Sciencedirect and AIS conference. Our search in the databases was conducted via the search string: ("crowd work*" OR "mass collaboration" OR "digital work" OR (crowdsourc* AND collaborat*)). This search string includes the logical OR operator, the logical AND operator and the free variable parameter*. The logical operators provide the correct relation among the substrings and the free variable parameter considers the string to be a substring of any other string, e.g. crowdsourc* considers crowdsourcing as well as crowdsource as well as crowdsourcees. With the logical AND operator crowdsourc* will be a match just in combination with collaborat*. With this string, a wide range of relevant literature is considered and literature with different focus than collaboration between crowdsourcees is excluded.

The literature selection process consists of three main steps. First, the search string has been searched for in title, keywords and abstract for gathering the literature from the databases. The restriction to "peer-reviewed" outlets was added as a condition to ensure a certain quality within the literature. The search covered January 2006 to February 2016, considering the rise of interest on crowdsourcing during that time [13]. In this step we found 547 publications with our search string. Second, by reviewing title, keywords and abstract of the 547 papers, we were able to reduce the amount to 136 by excluding not suitable papers. We excluded papers as not suitable that did not have any of the inflexions of the term collaboration and interaction accompanied by an inflexion of the word crowdsourcing or the terms "crowd work" and "digital work" in title, keywords or abstract. Most of the excluded papers had been selected by the search string, because the words "crowd" and "work" or "digital" and "work" were found in title, keywords and abstract separately from each other. Third, after a thorough analysis of the remaining 136 full texts, eight publications were considered for the study at hand. In this step, articles were excluded for the following reasons:

- no consideration of interpersonal interaction between crowdsourcees

- pure conceptual focus (e.g. defining phenomena)

- pure technological focus (e.g. deploying platforms)

- optimizations of algorithms/mechanism (e.g. automated task breakdowns or task incentive mechanism)

- task accomplishment in non collaborative work

- focus on interaction of different stakeholders (e.g. crowdsourcers and crowdsourcees) instead of interpersonal interaction among crowdsourcees.

Finally, the search was extended by analyzing the literature references of the final eight. No further literature was added at this point.

We use the selected papers as a basis to derive a model collaboration process among crowdsourcees, that consolidates recurring patterns from the papers.

\section{Overview of the considered literature}

Table 1 summarizes the considered literature and the result of our literature review.

First, we identified the specific domain addressed by each publication to and listed it under domain. The literature review reveals that studies addressing collaboration in a crowdsourcing context apply to different areas. For some publications a domain could not be identified, as those were dealing with crowdsourcing in general. According to Table 1, half of the considered literature [1], [3], [8], [15] and [22] is from the innovation domain and focus on idea and design contests.

This observation is in line with the extended need for collaboration in complex tasks described in the introduction of this paper, which is one potential reason for the prevalence of collaboration in the innovation domain. Other popular domains in crowdsourcing, such as e.g. crowdtesting are not represented in the literature we found. This finding 
gives indication, that collaboration may not have received substantial attention yet in these domains.

The collaboration topic is addressed differently in the publications. [1] address collaboration as part of a business model. The business model is a possible way for value creation. The collaboration process among crowdsourcees is described shortly. They suggest, that the crowd accomplishes tasks collaboratively and a selected subset of the crowd reviews the results and provides feedback or support for improvement [1]. Review and feedback are quality assurance mechanisms and also mentioned as an important step in [17].

[3] is focus on behavioral aspects that support the innovation level of contributions. They contribute guidelines to manage the crowd and move them towards innovation creation [3]. Their research provides information about the interaction among crowdsourcees by observing their behaviors.

[8] investigates the collaborative learning process in contests. In this setting, the collaboration is described first, when crowdsourcees try to find a

Table 1 Examined articles and the key findings

\begin{tabular}{|c|c|c|c|}
\hline $\begin{array}{c}\text { Author(s) and } \\
\text { year }\end{array}$ & Domain & Contribution & $\begin{array}{c}\text { Construct or findings } \\
\text { we adopted }\end{array}$ \\
\hline $\begin{array}{c}\text { Agafonovas and } \\
\text { Alonderiene } 2013 \\
{[1]}\end{array}$ & Idea contest & $\begin{array}{l}\text { They present a business model for successful } \\
\text { crowdsourcing. In terms of an empirical study of } \\
\text { crowdsourcing platforms in combination with expert } \\
\text { surveys, they examine the potential of value-creation in } \\
\text { this field and find structures within the crowd to ensure } \\
\text { qualitative task accomplishment. }\end{array}$ & $\begin{array}{l}\text { Quality assurance with } \\
\text { the concept of first and } \\
\text { second level users out of } \\
\text { the crowd. }\end{array}$ \\
\hline $\begin{array}{c}\text { Armisen and } \\
\text { Majchrzak } 2015 \text { [3] }\end{array}$ & Idea contest & $\begin{array}{l}\text { They conduct a empirical study to analyzing different } \\
\text { behaviors of crowdsourcees and their contributions to } \\
\text { characterize them. The characterizations are used to } \\
\text { derive guidelines for managing crowds. }\end{array}$ & $\begin{array}{l}\text { Interpretation of } \\
\text { communication patterns } \\
\text { among crowdsourcees. }\end{array}$ \\
\hline $\begin{array}{c}\text { Fantoni et al. } 2012 \\
\text { [7] }\end{array}$ & $\begin{array}{l}\text { Idea contest } \\
\qquad \& \\
\text { e-learning }\end{array}$ & $\begin{array}{l}\text { They present a collaborative learning process in idea } \\
\text { contests. In their empirical study, they analyze how to } \\
\text { learn through collaboration by studying the methods of } \\
\text { the winners in the idea contest. }\end{array}$ & $\begin{array}{l}\text { Interaction patterns } \\
\text { among crowdsourcees in } \\
\text { e-learning. Keeping and } \\
\text { passing knowledge } \\
\text { learned in a collaboration } \\
\text { process. }\end{array}$ \\
\hline $\begin{array}{l}\text { Haythornthwaite } \\
2009[10]\end{array}$ & $\begin{array}{c}\text { Other/ } \\
\text { undefined }\end{array}$ & $\begin{array}{l}\text { They present two models for collaborative activities. } \\
\text { Those models consider different attributes of the } \\
\text { crowdsourcing setting and distinguish between a loose } \\
\text { engagement level of crowdsourcees towards each other } \\
\text { and a closely related level of engagement. }\end{array}$ & $\begin{array}{l}\text { Considering engagement } \\
\text { level in the collaboration } \\
\text { process structure. }\end{array}$ \\
\hline $\begin{array}{l}\text { Hutter et al. } 2011 \\
\text { [13] }\end{array}$ & $\begin{array}{l}\text { Design } \\
\text { contest }\end{array}$ & $\begin{array}{l}\text { They have a behavior focus and study the effectiveness } \\
\text { of crowdsourcees with competitive and collaborative } \\
\text { behaviors compared to each other in design contests. } \\
\text { They conduct an empirical study to investigate how the } \\
\text { tension between those behaviors can influence the } \\
\text { quality of work. }\end{array}$ & $\begin{array}{l}\text { Interpretation of } \\
\text { communication patterns } \\
\text { among different behavior } \\
\text { types of crowdsourcees. }\end{array}$ \\
\hline $\begin{array}{c}\text { Kittur et al. } 2013 \\
{[15]}\end{array}$ & $\begin{array}{l}\text { Other/ } \\
\text { undefined }\end{array}$ & $\begin{array}{l}\text { They present a framework that considers different } \\
\text { aspects of the process of outsourcing to the crowd and } \\
\text { propose a potential structure for it. }\end{array}$ & $\begin{array}{l}\text { Quality assurance } \\
\text { structure. }\end{array}$ \\
\hline $\begin{array}{l}\text { Malhotra and } \\
\text { Majchrzak } 2014 \\
\text { [20] }\end{array}$ & Idea contest & $\begin{array}{l}\text { They use the knowledge integration process to manage } \\
\text { the crowd through collaborative idea contests. The } \\
\text { empirical study is used to derive management guidance } \\
\text { to navigate the crowd through the collaborative idea } \\
\text { finding process. }\end{array}$ & $\begin{array}{l}\text { Preparation } \quad \text { and } \\
\text { interpretation } \quad \text { of } \\
\text { communication patterns } \\
\text { among crowdsourcees. }\end{array}$ \\
\hline $\begin{array}{l}\text { Retelny et al. } 2014 \\
{[23]}\end{array}$ & $\begin{array}{c}\text { Other/ } \\
\text { undefined }\end{array}$ & $\begin{array}{l}\text { They present Flash teams as a possible way to } \\
\text { organize teams with experts from the crowd. The } \\
\text { empirical study tests the effectiveness of Flash teams } \\
\text { and shows the difference to none crowdsourced teams. }\end{array}$ & $\begin{array}{l}\text { Guidance towards the } \\
\text { collaboration product }\end{array}$ \\
\hline
\end{tabular}


solution for a problem announced by an open call and second, when a winner publishes the successful methods she/he used in the contest to solve the problem. By studying those methods, crowdsourcees learn and improve their skills as a part of the collaborative learning process. [12] presents the lightweight peer production (LWPP) and the heavyweight peer production (HWPP) model. The LWPP includes low level engagement among crowdsourcees. The collaboration process is loose in engagement, if the crowdsourcees show no dependency on interaction among each other and if they remain anonymous within the community. The HWPP model includes high engagement with a more intense connection between crowdsourcees [12]. These two

Table 2 Examined articles and their methodologies

\begin{tabular}{|c|c|c|c|c|c|c|c|c|}
\hline \multirow[t]{2}{*}{$\begin{array}{c}\text { (Author(s) and } \\
\text { year) }\end{array}$} & \multicolumn{5}{|c|}{$\begin{array}{c}\text { Research } \\
\text { approaches }\end{array}$} & \multicolumn{3}{|c|}{$\begin{array}{c}\text { Type of } \\
\text { contribution }\end{array}$} \\
\hline & 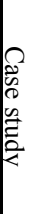 & 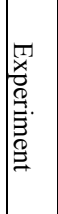 & 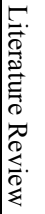 & 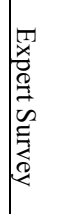 & 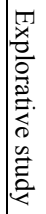 & 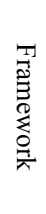 & $\begin{array}{l}3 \\
\stackrel{3}{0} \\
0\end{array}$ & 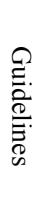 \\
\hline $\begin{array}{c}\text { Agafonovas and } \\
\text { Alonderiene } 2013\end{array}$ & $\mathrm{x}$ & & & $\mathrm{x}$ & & & $\mathrm{x}$ & \\
\hline $\begin{array}{c}\text { Armisen and } \\
\text { Majchrzak } 2015\end{array}$ & $\mathrm{x}$ & & & & & & & $\mathrm{x}$ \\
\hline Fantoni et al.2012 & $\mathrm{x}$ & & & & & $\mathrm{x}$ & & \\
\hline $\begin{array}{c}\text { Haythornthwaite } \\
2009\end{array}$ & & & & & & & $\mathrm{x}$ & \\
\hline Hutter et al. 2011 & & & $\mathrm{x}$ & & $\mathrm{x}$ & & & $\mathrm{x}$ \\
\hline Kittur et al. 2013 & & & & $\mathrm{x}$ & & $\mathrm{x}$ & & \\
\hline $\begin{array}{c}\text { Malhotra and } \\
\text { Majchrzak } 2014\end{array}$ & & $\mathrm{x}$ & & $\mathrm{x}$ & & & & $\mathrm{x}$ \\
\hline Retelny et al. 2014 & $\mathrm{x}$ & & & & & $\mathrm{x}$ & & \\
\hline
\end{tabular}

models distinguish two types of engagement levels each suited for different domains [12]. They describe interactions we can use for the collaboration process. [17] introduces a framework for crowdwork. The framework structures the workforce of the crowdsourcees to be functional and controllable to ensure comparability with organizational teams.

[22] guides the crowdsourcees through the collaborative idea contest by providing instructions and operating with the knowledge integration process. Their results are guidelines for managing the crowd. Their adaption of the knowledge integration process delivers interaction patterns. [24] introduces Flash teams as a possible way to structure teams. This framework considers different concepts, as for e.g. distributed leadership [21] to ensure better team performance [24]. They describe the interaction between team members out of their conducted case study.

[15] found that in a contest environment crowdsourcees are more successful by being collaborative and slightly competitive.

According to Table 1, the authors demonstrate different research focus on the crowdsourcing domain and they treat different aspects in their research. Table 2 . delivers an overview of the the research methods and the three following types of contributions of the considered literature. We differentiate between framework as abstraction of an approach, model as representation of a phenomenon [9] and guidelines as recommendations that should be considered in concepts. According to Table 2, [12] contributes the LWPP and the HWPP models by arguing about individuals and the collaborative connection to each other.

[17] derives a framework based on the results of expert surveys from an enterprise perspective [17]. This approach is similar in its goal with the approach of [24], but they differ in the research methods they used. The results of [24] are based on a case study. [1] used two methods. They conducted a case study and used expert surveys. The scope of their study is how to run crowdsourcing initiative. One important aspect in their effort is to point out the relevance of human capital for running crowdsourcing initiatives. Here they enter the collaboration topic and understand it as a part of human capital. The result of the case study points out the relevance of collaboration as a key to access intellectual resources for value creation. Their main contribution is a business model. They consider the knowledge flow in crowdsourcing initiative and the whole network of stakeholders to derive the business model [1]. The research method case study is used more than any other in the considered literature. [3] conducted a case study as well as [8], but they differ in the type of contribution. While [8] present a framework for the collaborative learning process in e-learning, [3] contribute guidelines they derive based on the case study by analyzing the behaviors of individuals. More guidelines are delivered by $[15,22]$, but both publications show different research methods. The research of [22] based on an experiment in combination with an expert survey and [15] conduct an explorative study after a literature review.

Summarizing the findings, the analysis of the literature shows many studies and less theoretical approaches. 
This indicates a current research interest and that it is still in the exploration phase.

\section{Status-quo}

Most considered papers do not present a collaboration process among crowdsourcees. However, the knowledge integration process [11] is used to guide the crowdsourcees through the process of idea contests

\begin{tabular}{|c|c|c|}
\hline \multicolumn{3}{|c|}{ Knowledge Integration Process } \\
\hline Sharing & Highlighting & Combining \\
\hline $\begin{array}{l}\text { - Posting Ideas, examples, } \\
\text { facts, tradeoffs }\end{array}$ & $\begin{array}{l}\text { Voting on others } \\
\text { posts }\end{array}$ & $\begin{array}{l}\text { - Creating solutions } \\
\text { by comibing }\end{array}$ \\
\hline - commenting & $\begin{array}{l}\text { - Promoting } \\
\text { others'comments }\end{array}$ & $\begin{array}{l}\text { knowledge in } \\
\text { multiple posts }\end{array}$ \\
\hline
\end{tabular}

Figure 1. Knowledge integration process [22]

[22]. From a collaboration perspective the knowledge integration process summarizes aspects of interactions between crowdsourcees. The knowledge integration process in its current state is limited to the innovation domain. In innovation contests the crowdsourcees need to post new contributions or comment on contributions $[15,22],[3,8]$. The adaption of the knowledge integration process for idea contests consist of three main phases [22]. According to Figure 1, those phases are the sharing phase, the highlighting phase and the combining phase. In the sharing phase, the crowdsourcees contribute new ideas through posts or they add new aspects to the contributions of others with comments. The purpose is to widen the range of ideas and view them from different perspectives. The highlighting phase is for the crowdsourcee to point out the favored contributions and support them via votes or comments. Finally, in the combining phase crowdsourcees need to consider the contributions of others from previous phases and start to filter and connect the knowledge to consolidate solutions to be ready for submitting [22].

This adaption of the knowledge integration process serves to structure the crowdsourcees interactions with each other and can be useful as a base to derive indicators for a more general collaboration process among crowdsourcees.

\section{Collaboration process}

The understanding of the collaboration process among crowdsourcees is not yet fully addressed in the current state of research, as the low number of previous publications indicates. In our attempt to derive a representative collaboration process, we analyzed the content of each paper included and searched for indication for process phases. We found three main phases. Each phase consists of more than one step or sub-phase.

The steps result from our analysis of activities mentioned in the literature. Table 3 delivers an overview of the phases and the assigned publications, which contains indications for step of respective phase.

The introduction and discussion of the steps will be presented in the following subsections of this section. The subsections will be structured by the following phases. First there is a phase before the crowdsourcees collaborate. We call this phase the Pre Collaboration Phase. Pre stands for preparation and describes the phase in which crowdsourcees take preparations to be ready to collaborate with each other. This phase is affected by individual efforts of crowdsourcees, which can have qualitative impact on the collaboration.

Table 3. Content analysis of the Literature

\begin{tabular}{|c|c|c|}
\hline Phases & Steps & Literature \\
\hline \multirow{4}{*}{$\begin{array}{c}\text { Pre } \\
\text { Collaboration } \\
\text { Phase }\end{array}$} & Motivation & [17] \\
\hline & Instruction & {$[14,21,23]$} \\
\hline & $\begin{array}{c}\text { Shared } \\
\text { understanding }\end{array}$ & {$[3,8,14,21,23]$} \\
\hline & Participation & [21] \\
\hline \multirow{4}{*}{$\begin{array}{l}\text { Collaboration } \\
\text { Process }\end{array}$} & Prototyping & {$[1,3,8,14,21,23]$} \\
\hline & Feedback & {$[1,3,8,15,17,22,24]$} \\
\hline & Revise & {$[1,15,17,22,24]$} \\
\hline & Submit & {$[3,8,15,22,24]$} \\
\hline \multirow{3}{*}{$\begin{array}{c}\text { Post } \\
\text { Collaboration } \\
\text { Phase }\end{array}$} & $\begin{array}{c}\text { Process } \\
\text { Documentation }\end{array}$ & {$[8,24]$} \\
\hline & $\begin{array}{c}\text { Sharing } \\
\text { knowledge/methods }\end{array}$ & [8] \\
\hline & Learning & [8] \\
\hline
\end{tabular}

Second there is the actual Collaboration Process. This phase includes the interaction between crowdsourcees and follows a general collaboration process based on the current state of the literature.

Last, there is the phase after the collaboration between the crowdsourcees. We call this phase the Post Collaboration Phase and it represents activities that can influence further collaboration attempts.

\subsection{Pre Collaboration Phase}

Before the main collaboration process between crowdsourcees begins, the literature mentions aspects that can influence the collaboration process. These aspects are based on individuals. These steps serve as optimal preparation for entering the collaboration process. There are four steps, that need to be 
considered. The Motivation, Instruction, Shared Understanding and the Participation step.

5.1.1 Motivation. There are motivational aspects for crowdsourcees to participate in the collaboration process. "Motivating workers to accomplish such tasks can be challenging, and may lead to reduced engagement with the system" [17]. Attracting and motivating crowdsourcees is important to increase their interest in the collaborative task and shows qualitative impact on the outcome $[2,10,16,20,25,27]$.

5.1.2 Instructions. A crowdsourcer needs to consider giving instructions for participation in an open call. This step helps crowdsourcees to get better navigation through the process of participation. Instructions could contain specific guidelines for contributing [22]. Specific structures and methods to publish knowledge help both the contributor and other crowdsourcees to get a picture of the idea.

Instructions can consist of deadlines for special submissions, reviews or activities. In [15] different time phases are described, when crowdsourcees have different responsibilities towards tasks. The right relation between input and output of a task is also a relevant [24] set of instructions.

5.1.3 Shared Understanding. Shared understanding is a step for crowdsourcees to understand each other and ensures to perceive the right knowledge from contributions. After reacting to an open call the crowdsourcees need to bring themselves up to date. The literature about innovation contests describes comments on posts $[3,8,15,22]$, "comments on comments" [3] or "replies to replies" [13] and literature from other domains mentions documentation for e.g. task descriptions [24]. This means, crowdsourcees need to look up, read, embed, evaluate and understand the contributions by others. This may sound trivial, but is an important step to avoid duplicates and misunderstandings. The shared understanding step ensures to acquire the knowledge needed for being qualified to contribute in the collaboration process. If crowdsourcees gather an overview of the contributions others made, there are different options they have. If something is unclear, then the crowdsourcee can ask questions via comments. If knowledge is missing to understand the contribution, then research must be done to acquire the missing knowledge for e.g. considering documentations added by others. When everything is clarified and the contributions by others are understood, the crowdsourcees can work on own contributions by extending others or creating new contributions [15,22], [3] .
5.1.4 Participation. The last step in the Pre Collaboration Phase is Participation. The crowdsourcees follow the Instructions to create a contribution. This can be posting ideas $[3,22]$, creating designs [15], solving problems [8], accomplishing tasks [24] or commenting on others' contribution $[15,22]$. Open questions discovered in the shared understanding step can be constructed as comments. In addition, replies to the comments would help to answer the questions. The choice of contribution depends on the content of the contributions and decides the kind of participation of the crowdsourcees.

\subsection{Collaboration Process Phase}

The Collaboration Process Phase consists of the main collaboration process among crowdsourcees. The collaboration of crowdsourcees results in an outcome, that serves to accomplish the tasks of an open call.

The knowledge base, we can use to build on, is delivered through the adaption of the knowledge integration process [22] according to Figure 1. However, we found indication in the papers that other, more complex crowdsourcing tasks might require a more detailed process structure than the knowledge integration process proposes. The goal in idea contests is to collaboratively produce an innovative idea to solve a problem described in an open call. To do so, the crowdsourcees first create artefacts of solutions, by adding their ideas or new aspects to the ideas that already have been posted [22], according to the sharing phase of Figure 1. We observed different types of artefacts in the considered literature as there were early design versions [15], sketches, mockups and early versions of incomplete system [24]. These artefacts are the first milestone the collaboration among crowdsourcees achieves and serve as a base to work on. The second milestone is to reflect and communicate the deficits of the artefact and emphasize improvements $[1,8,17,24]$. The activities of this milestone can be categorized as the activities between the sharing and highlighting phase of the knowledge integration process. The highlighting of deficits can be done with comments and promotion of those comments similar to the commenting part of the sharing phase and the promoting of the highlighting phase.

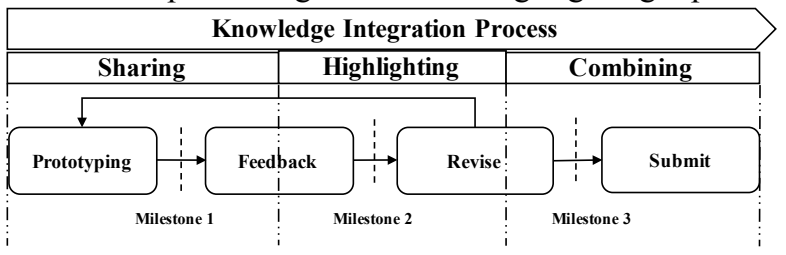

Figure 2. Collaboration process

The last milestone before submitting the outcome of the collaboration, is the step to consider the 
suggested improvements and build them in the artefact to produce progress, that can result in an end product $[1,17,24]$. The last milestone refers to a combination of the highlighting phase and the combining phase in the knowledge integration phase. The voting activity of the highlighting phase reflects the improvements and collaboratively leads to decide if the improvement needs to be considered in further elaboration of the artefact, which is an activity of the combing phase.

The discussion above leads us to derive the collaboration process according to Figure 2. The collaboration process consists of the four process steps prototyping, feedback, revise and submit. We assigned the relation between the collaboration process and the knowledge integration process in Figure 2. Furthermore, we visualized the three milestones within also in Figure 2. The steps of the collaboration process will be discussed in the following subsections of this section.

5.2.1 Prototyping. The goal of this step is to produce an artefact collaboratively. To produce such an artefact "[...] each product goes through two stages brainstorming among the first level users and elaboration of the selected ideas [...]" [1]. First level users in this context are crowdsourcees. This indicates two collaborative interactions. First is the brainstorming between crowdsourcees and the second is to elaborate on the results of the chosen ideas. Brainstorming consists of two main aspects, the idea presentation and their extension. This is described in the innovation domain as "submitted ideas combined with a large number of outgoing as well as incoming comments" [15]. However, elaboration follows afterwards and consists of creating the first prototype. Creating prototypes can be done by "prototyping many ideas in parallel leads" [24] or the creation needs to be "mock up interface alternatives" [17], but more relevant steps may be needed such as "a usability analyst to test those prototypes, and a front-end engineer to implement the best one" [17]. The outcome of the prototype depends on the task and the domain. In innovation contests the outcome can be designs [15] or ideas for a solution, that solve a problem $[1,3,8,22]$.

We derive the following interactions between crowdsourcees in this process step:

1. Brainstorming

2. Elaboration
a. Idea presentation
b. Idea extension

a. Limit the numbers of ideas

b. Create prototype(s) considering the chosen ideas
When this process step is accomplished, the first milestone is reached and the crowdsourcees can strive to reach the next one.

5.2.2 Feedback. This process step has a dependency on the previous one, by needing the prototypes to gather different kind of feedback from different sources e.g. "[...]taking heuristic evaluation report [...]" [24] or crowdsourcees "[...] provide feedback, and answer questions through the chat [...]" [24]. The questions and answers are indications that the crowdsourcees interact to understand how the prototype serves to accomplish the task. Afterwards the heuristic evaluation part checks the prototype for deficits. If these steps are accomplished, crowdsourcees can consider, that "incoming comments also provide a lot of feedback, knowledge and suggestions, and allow these users to benefit from the opportunity to collaborate in the creative process in order to enhance and perfect their individual ideas" [15]. This indicates that, if the deficits are identified, the crowdsourcees discuss improvements and deliver suggestions or new ideas for fixing the deficits.

We identify the following interactions among crowdsourcees in this process step:

1. Discuss prototype and its value for task accomplishment.

2. Check for deficits and highlight them.

3. Suggest improvements.

With this interaction patterns the second milestone will be reached.

5.2.4 Revise. This process step combines the results of the previous process steps to consider the suggested improvements when further developing the prototype. This is reflected in "[...] Revise a mockup based on feedback." [24] and "[...] using suggestions of the ideas selected by the core team" [1]. The core team consists of selected members from the crowd, who provide feedback on the work produced by the crowd [1]. The crowdsourcees need to go through the feedbacks and filter the relevant aspects and suggestions before considering them for the prototype. The development of the prototype to a further version can be analogical to "User " $A$ " formulated his idea, which is the key contribution toward the final idea proposed by user "B"' [8]. We can observe that the idea of $A$ is the prototype and user $B$ induces his improvement suggestions into the prototype, which results in an end product. If this is not possible because the feedback leads to a new concept of a new prototype that is independent of the current prototype, this process step suggests a loopback to the first process step and to construct a new prototype. "This rapid, 
parallel cycle could allow end users to quickly iterate toward high-quality ideas" [24].

We derive the following interaction patterns for this process step:

1. Filtering feedback for relevant improvements

2. Development of the prototype by considering the filtered improvement suggestions

or

1. Filtering feedback for relevant improvements

2. Use the loopback to process step one (Prototyping)

After filtering the feedback, the crowdsourcees can advance the prototype towards the end product and reach the final milestone or they decide to use the loopback and take the feedback to construct a new prototype. In this case, the final milestone could not be reached and they start fresh to reach the first milestone. But in the first step they don't need to start with the brainstorming part. They can skip this part, because they already have elaborated on the concept for a new prototype by working through the feedback.

6.2.1 Submit. This process step is entered if an end product is produced. This means all three milestones are reached. This process step covers instructions for submitting the end product. The instructions may consist of defined types of the end product [24]. Those can be formulated concepts of ideas structured and explained in text $[1,3,8,22]$ (e.g. documents), visually constructed designs [15], the source code of a (mobile) application [24], etc. This step checks for and converts the end product to meet prerequisites for submitting. Finally, the crowdsourcees can submit and the collaboration process ends.

\subsection{Post Collaboration phase}

This phase starts after completion of the collaborative task and begins with the process documentation. This phase serves to prepare follow up future collaboration processes and to benefit from the experience of the accomplished collaboration process.

5.3.1 Process documentation. Usually, important knowledge used to accomplish the collaborative task is documented on the crowdsourcing platform, e.g. in chats, mails or a ticket system. Crowdsourcing platforms in the innovation (contest) domain often visualize the contributions of the participants for the crowdsourcees. An example of it is the history of the contributions such as described in $[3,8,15,22]$. The documentations in other domains are descriptions of the tasks in combination with the documents of the collaborative activities that relate to the task $[8,17,24]$.
It is important to filter relevant knowledge and make it available for use in future similar tasks [8].

5.3.2 Sharing Knowledge/Methods. The next topic discussed by [8] is to pass the knowledge and the successful methods, used to accomplish a task, to the crowdsourcing community. This can be used by crowdsourcees in the shared understanding step of the Pre Collaboration phase. The process documentation helps to identify the key elements of methods towards optimal task accomplishment. Sharing them with the community helps to use the gathered experience from project to project and may lead not just to the progress of the individual but the whole community [8].

5.3.3 Learning. The last topic in this phase is for a crowdsourcee to learn from the experience. This means to compare own and the shared knowledge and methods by others with each other. The goal is to identify important methods and add them to the own repertoire and get back to them in new open calls [8].

\section{Framework Overview}

The framework according to Figure 3 combines and structures the results from the previous section. We introduce this framework as a first suggestion for a model collaboration process in the crowdsourcing field. The framework considers the phases before and after the collaboration of the crowdsourcees and suggests a collaboration process design for the interaction between the crowdsourcees with the goal to accomplish tasks collaboratively.

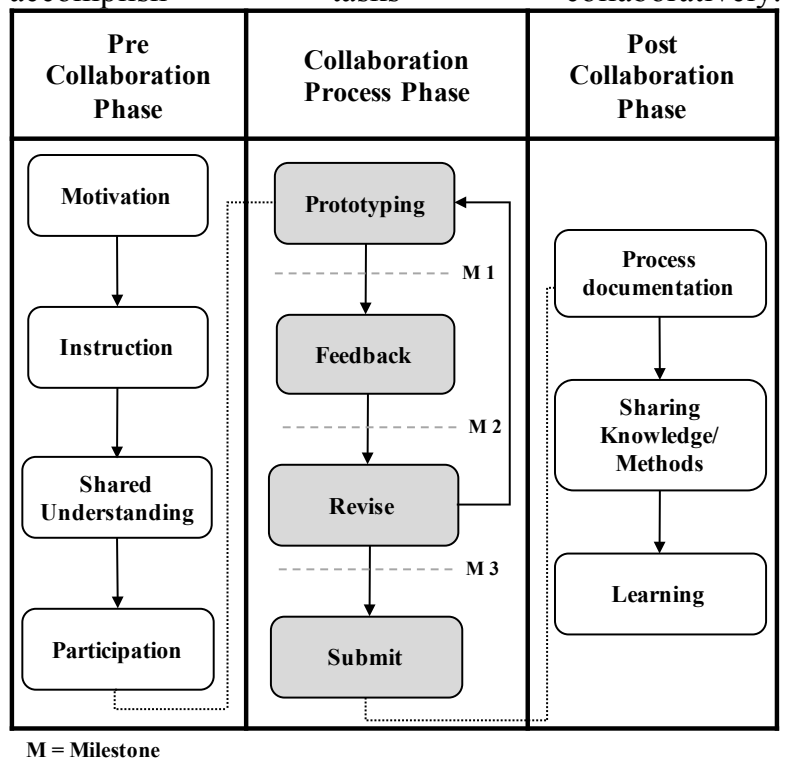

Figure 3. Collaboration process design framework for crowdsourcing 
According to Figure 3, the framework consists of the Pre Collaboration Phase, Collaboration Process Phase and the Post Collaboration Phase. The Pre Collaboration Phase considers the individual effort of crowdourcees for taking preparation for the collaborative work. This includes the crowdsourcee to be motivated, consider the instructions for participating and build a knowledge base through shared understanding to be ready for optimal participation. shall e.g. be applied to identify, in which process steps what kind of problems occur and how they can be solved. This can serve to optimize collaboration processes and control the efficiency of the interaction among crowdsourcees.

Crowdsourcing initiatives can adopt or use the framework as guidance to establish features on platforms to control and support the collaboration among crowdsourcees.

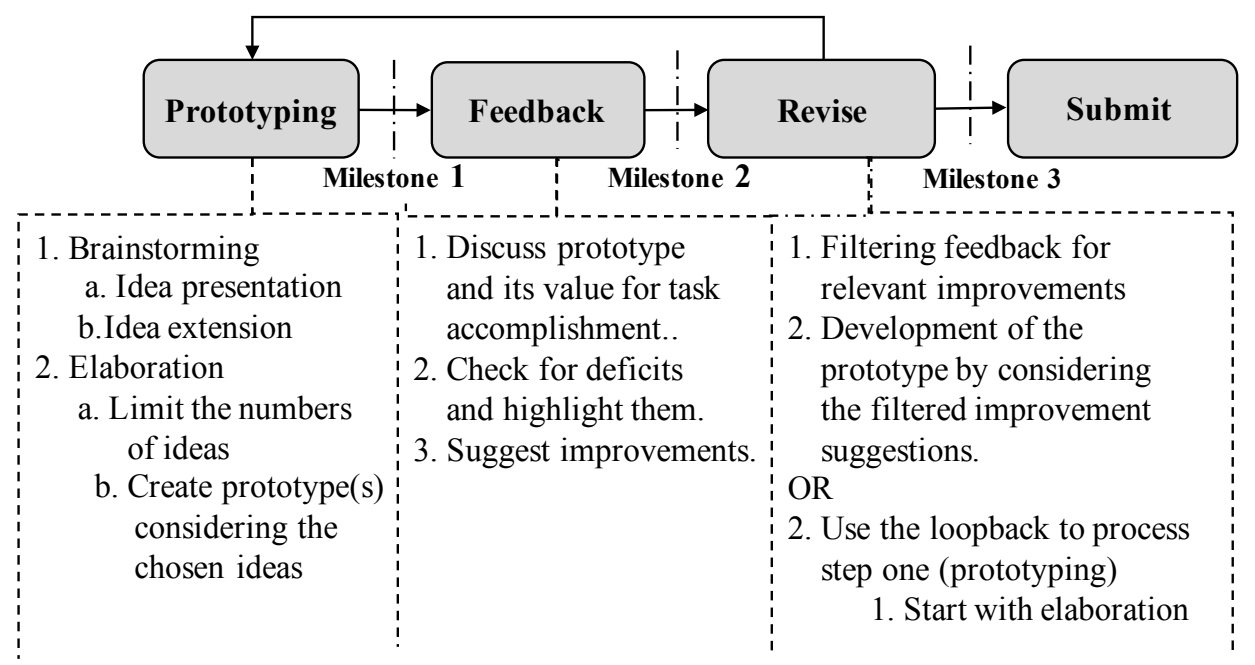

Figure 4. Detailed view of the Collaboration process phase

The Collaboration Process Phase consists of the main collaboration process design for the interaction among crowdsourcees. A detailed view with description of the four steps is presented in Figure 4. Finally, the Post Collaboration phase defines procedures to capture the experience from the collaboration process and to develop new skills by first documenting the process, second sharing the knowledge and methods, that were used in the collaboration process and third to learn from the shared knowledge and methods of the others.

\section{Conclusion}

The presented framework in this paper contributes to a rising area of interest in research and practice by gathering the current state of the research and deriving knowledge on collaboration among crowdsourcees out of the literature.

The framework can serve as a basis to design structured collaboration processes among crowdsourcees in the crowdsourcing field. Moreover, the framework can be used to identify the current state of a running process on a crowdsourcing platform or to analyze completed collaboration processes for weaknesses in the interaction and problem identification in different scenarios. The framework
This paper grounds the presented framework on the current state of knowledge from the crowdsourcing field and addresses the specific demands of crowdsourcing. Future research shall check if the presented framework can be adopted to other research domains or if it can be developed further with insights from related domains, e.g. the open innovation field. Moreover, future research shall validate the framework and iteratively advance it. In particular, it should, explore the real world interaction among crowdsourcees with the use of the framework. On this way, this paper makes a contribution towards a better understanding and well informed design of crowdsourcing collaboration process.

\section{References}

[1] Agafonovas, A. and Alonderiene, Raimonda. Value Creation in Innovations Crowdsourcing: Example of Creative Agencies. Organizations and Markets in Emerging Economies 2, 1(7) (2013).

[2] Arakji, R.Y. and Lang, K.R. Digital Consumer Networks and Producer - Consumer Collaboration: Innovation and Product Development in the Video Game Industry. $J$. of Management Information Systems 24, 2 (2007), 195-219. 
[3] Armisen, A. and Majchrzak, A. Tapping the innovative business potential of innovation contests. Business Horizons 58,4 (2015), $389-399$.

[4] Bittner, E.A.C. and Leimeister, J.M. Creating Shared Understanding in Heterogeneous Work Groups: Why It Matters and How to Achieve It. J. of Management Information Systems 31, 1 (2014), 111-144.

[5] Bowers, C.A., Pharmer, J.A., and Salas, E. When member homogeneity is needed in work teams a meta-analysis. Small group research 31, 3 (2000), 305-327.

[6] Brocke, J. vom, Simons, A., Niehaves, B., Riemer, K., Plattfaut, R., and Cleven, A. Reconstructing the Giant: On the Importance of Rigour in Documenting the Literature Search Process. Proceedings of the $17^{\text {th }}$ European Conference on Information Systems, (2009).

[7] Durward, D., Blohm, I., and Leimeister, J.M. Crowd Work. Business \& Information Systems Engineering 58, 4 (2016), 281-286.

[8] Fantoni, G., Apreda, R., Gabelloni, D., and Montelisciani, G. You solve, I learn: A novel approach to e-learning in collaborative crowdsourcing. Engineering, Technology and Innovation (ICE), 2012 18th International ICE Conference on, (2012), 1-10.

[9] Frigg, R. and Hartmann, S. Models in Science. In E.N. Zalta, ed., The Stanford Encyclopedia of Philosophy. 2012.

[10] Gloor, P.A. and Cooper, S.M. The New Principles of a Swarm Business. MIT Sloan Management Review 48, 3 (2007), 81-84.

[11] Grant, R.M. Prospering in Dynamically-Competitive Environments: Organizational Capability as Knowledge Integration. Organization Science 7, 4 (1996), 375-387.

[12] Haythornthwaite, C. Crowds and Communities: Light and Heavyweight Models of Peer Production. in Proceedings of the 42nd HICSS, (2009), 1-10.

[13] Howe, J. The Rise of Crowdsourcing. Wired Magazine 14, http://www.wired.com/wired/archive/14.06/crowds.html (timestamp: 2008-12-21), 6 (2006).

[14] Howe, J. Crowdsourcing: Why the Power of the Crowd is Driving the Future of Business. Crown Business, New York, 2008.

[15] Hutter, K., Hautz, J., Füller, J., Mueller, J., and Matzler, K. Communitition: The Tension between Competition and Collaboration in Community-Based Design Contests. Creativity and Innovation Management 20, 1 (2011), 3-21.

[16] Kaufmann, N., Schulze, T., and Veit, D.J. More than fun and money. Worker Motivation in Crowdsourcing - A Study on Mechanical Turk. Proceedings of the 17th Americas Conference on Information Systems (AMCIS 2011), AISeL (2011), Paper 340.

[17] Kittur, A., Nickerson, J.V., Bernstein, M., et al. The Future of Crowd Work. Proceedings of the 2013 Conference on Computer Supported Cooperative Work, ACM (2013), 1301-1318.

[18] Langan-Fox, J., Anglim, J., and Wilson, J.R. Mental models, team mental models, and performance: Process, development, and future directions. Human Factors and Ergonomics in Manufacturing 14, 4 (2004), 331-352.

[19] Leimeister, J.M. Mitbestimmung. Hans Bökler Stiftung, 02/2016 (2016), 20-24.

[20] Leimeister, J.M., Huber, M., Bretschneider, U., and Krcmar, H. Leveraging Crowdsourcing: ActivationSupporting components for IT-based ideas competition. Journal of Management Information Systems (JMIS) 26, 1 (2009), 197-224.

[21] Luther, K., Fiesler, C., and Bruckman, A. Redistributing Leadership in Online Creative Collaboration. Proceedings of the 2013 Conference on Computer Supported Cooperative Work, ACM (2013), 1007-1022.

[22] Malhotra, A. and Majchrzak, A. Managing Crowds in Innovation Challenges. California Management Review 56, 4 (2014), 103-123.

[23] Pedersen, J., Kocsis, D., Tripathi, A., et al. Conceptual Foundations of Crowdsourcing: A Review of IS Research. System Sciences (HICSS), 2013 46th Hawaii International Conference on, (2013), 579-588.

[24] Retelny, D., Robaszkiewicz, S., To, A., et al. Expert Crowdsourcing with Flash Teams. Proceedings of the 27th Annual ACM Symposium on User Interface Software and Technology, ACM (2014), 75-85.

[25] Wagner, C. and Prasarnphanich, P. Innovating Collaborative Content Creation: The Role of Altruism and Wiki Technology. Proceedings of the 40th Annual Hawaii International Conference on System Sciences, IEEE Computer Society (2007), 18 b.

[26] Wegge, J.; Roth, c.; Neubach, B.; Schmidt, K.-h.; and Kanfer, r. Age and gender diversity as determinants of performance and health in a public organization: the role of task complexity and group size. Journal of Applied Psychology 93, 6 (2008), 1301-1313.

[27] Yu, L.L. and Nickerson, J.V. Generating creative ideas through crowds: An experimental study of combination. Thirty Second International Conference on Information Systems, (2011). 